\title{
Nature of the $f_{0}(600)$ Scalar Meson from its $N_{c}$ Dependence at Two Loops in Unitarized Chiral Perturbation Theory
}

\author{
J. R. Peláez and G. Ríos \\ Departamento de Física Teórica II, Universidad Complutense de Madrid, 28040 Madrid, Spain
}

(Received 25 August 2006; published 12 December 2006)

\begin{abstract}
By using unitarized two-loop chiral perturbation theory partial waves to describe pion-pion scattering we find that the dominant component of the lightest scalar meson does not follow the $\bar{q} q$ dependence on the number of colors that, in contrast, is obeyed by the lightest vectors. The method suggests that a subdominant $\bar{q} q$ component of the $f_{0}(600)$ possibly originates around $1 \mathrm{GeV}$.
\end{abstract}

DOI: 10.1103/PhysRevLett.97.242002

The lightest scalar mesons are a controversial subject that is receiving relevant contributions that could help settle questions about their existence and nature. Experimentally, several analyses [1] find poles for the $f_{0}(600)$ and $\kappa$, the lightest scalars with isospin 0 and $1 / 2$, respectively. The former is of interest for spectroscopy but also for understanding spontaneous chiral symmetry breaking, since it has the vacuum quantum numbers. Theoretically, the QCD chiral symmetry breaking pattern leads to $f_{0}(600)$ and $\kappa$ poles in $\pi \pi$ and $\pi K$ scattering [26]. Concerning the spectroscopic classification, most hadronic models cannot extract the quark and gluon composition without assumptions hard to justify within QCD. In contrast, using fundamental degrees of freedom, i.e., in lattice or with QCD inspired potentials, leads to problems related to chiral symmetry breaking, physical masses of quarks and mesons, or decay constants. All approaches are also complicated by possible mixings of different states in the physical one. Most of these caveats are overcome in an approach [7] ([8] for a review) based on the pole dependence on the number of colors $N_{c}$ of meson-meson scattering within unitarized chiral perturbation theory (ChPT).

The relevance of the large $N_{c}$ expansion [9] is that it provides an analytic approach to QCD in the whole energy region and a clear identification of $\bar{q} q$ states that become bound as $N_{c} \rightarrow \infty$, and whose masses scale as $O(1)$ and their widths as $O\left(1 / N_{c}\right)$. Other hadronic states may show different behaviors [10].

In order to avoid any spurious $N_{c}$ dependence in the hadronic description we use ChPT, namely, the QCD low energy effective theory, where the large $N_{c}$ behavior is implemented systematically. It is built as the most general derivative expansion of a Lagrangian [11], in terms of $\pi$, $K$, and $\eta$ mesons compatible with the QCD symmetries. These particles are the Goldstone bosons associated to the spontaneous chiral symmetry breaking of massless QCD and are therefore the lightest degrees of freedom. Actually, the $u, d$, and $s$ quark masses are nonvanishing but small enough to be treated as perturbations that give rise to $\pi, K$, and $\eta$ masses. Thus, ChPT is an expansion in powers of momenta and masses and its applicability is limited to a few hundred $\mathrm{MeV}$ above threshold. Each order is made of
PACS numbers: 12.39.Mk, 11.15.Pg, 12.39.Fe, 13.75.Lb

all possible terms multiplied by a "chiral" parameter. These low energy constants (LECS) are renormalized to absorb loop divergences order by order, and once determined from experiment they can be used in any other pseudo Goldstone boson amplitude.

Here we are interested in meson-meson scattering since resonances not initially present in ChPT can be generated dynamically by unitarization $[4-6,12-14]$. Indeed $[8,14]$, with the coupled channel inverse amplitude method (IAM), the one-loop ChPT meson-meson amplitudes describe data up to $\sqrt{s} \simeq 1.2 \mathrm{GeV}$ and generate the $\rho$ and $K^{*}$ vectors, and the $f_{0}(600), \kappa, a_{0}(980)$, and $f_{0}(980)$ scalars, using LECS compatible with standard ChPT and therefore without any further assumption or source of spurious $N_{c}$ dependence.

By scaling the one-loop ChPT parameters with their $N_{c}$ behavior, it was shown that the generated $\rho$ and $K^{*}$ show the typical $N_{c}$ behavior of $\bar{q} q$ states, whereas the scalars are at odds with a dominant $\bar{q} q$ component. These results, confirmed by other methods [15], implied some cancellation between tree level diagrams proportional to LECS, and that loop diagrams with two intermediate mesons are very relevant in the generation of light scalars. But such loop diagrams are subdominant in the $1 / N_{c}$ counting and one could wonder about the stability under small changes in the LECS and about higher order ChPT corrections that could become larger than loop terms at sufficiently large $N_{c}$, and reveal a subdominant $\bar{q} q$ component.

Here we present a method to quantify the above statements, and generalize the approach of [7] to two-loop ChPT $\pi \pi$ scattering [16]. Despite the many second order parameters and their large uncertainties, data can be well described and we find again that the $\rho(770)$ behaves as $\bar{q} q$ with $N_{c}$, whereas the $f_{0}(600)$ main component does not. Furthermore, with the second order calculation a dominant $\bar{q} q$ behavior cannot be imposed on the $f_{0}(600)$ and the $\rho$ simultaneously, but a subdominant $\bar{q} q f_{0}(600)$ component seems to arise at larger $N_{c}$ around $1 \mathrm{GeV}$.

At leading order, the only parameter is the pion decay constant in the chiral limit, $f_{0}=O\left(\sqrt{N_{c}}\right)$, fixed by the spontaneous symmetry breaking scale $4 \pi f_{0} \simeq 1 \mathrm{GeV}$. Indeed, ChPT $\pi \pi$ scattering amplitudes are expanded as $t \simeq t_{2}+t_{4}+t_{6}+\ldots$ with $t_{k}=O\left[\left(p / 4 \pi f_{0}\right)^{k}\right]$ and are, 
generically, $O\left(1 / N_{c}\right)$. In particular, the LECS appearing in $\pi \pi$ scattering at $O\left(p^{4}\right)$ [11] scale as $O\left(N_{c}\right)$. We use the $S U$ (2) notation, $l_{i}^{r}$, since we are only dealing with $\pi \pi$ scattering (see the last reference in [11] for a translation to $S U(3)$ ). In Table I we provide a sample of $l_{i}^{r}$ sets given in the literature, whose differences we take as systematic uncertainties for the set we use in our fits below. In Table II we list the six $O\left(p^{6}\right)$ constants appearing in $\pi \pi$ scattering, denoted $r_{i}$, that count as $O\left(N_{c}^{2}\right)$. Those values are estimates assuming they are saturated by the lightest resonances. This hypothesis works well at $O\left(p^{4}\right)$ [20], but for $O\left(p^{6}\right)$ is likely correct within an order of magnitude [16] so we assign a $100 \%$ uncertainty.

The $1 / N_{c}$ counting does not specify at what renormalization scale $\mu$ it applies, which is an uncertainty studied in [7] for the one-loop LECS. For the $r_{i}$, the scale dependence is more cumbersome and has not been written explicitly. Nevertheless, in both cases it is subleading in $1 / N_{c}$, and since we have a $100 \%$ error on the $r_{i}$, it should be well within errors for our fits. Hence, we do not perform such analysis here, setting $\mu=770 \mathrm{MeV}$, as usual.

Next, resonances can be found as poles in partial wave amplitudes $t_{I J}$ of isospin $I$ and angular momentum $J$ that, in the elastic regime satisfy the unitarity condition

$$
\operatorname{Im} t=\sigma|t|^{2} \Rightarrow \operatorname{Im} \frac{1}{t}=-\sigma \Rightarrow t=\frac{1}{\operatorname{Re} t^{-1}-i \sigma},
$$

where $\sigma$ is the two-meson phase space and we have omitted the $I J$ indices for brevity. Note that ChPT expansions violate exact unitarity, since in the first Eq. (1), the highest power of momenta on the right hand is twice that on the left. Unitarity is only satisfied perturbatively

$$
\operatorname{Im} t_{2}=0, \quad \operatorname{Im} t_{4}=\sigma t_{2}^{2}, \quad \operatorname{Im} t_{6}=\sigma 2 t_{2} \operatorname{Re} t_{4} .
$$

If we replace in Eq. (1) $\operatorname{Re} t^{-1}$ by its ChPT approximation we get the IAM that satisfies elastic unitarity exactly. At $O\left(p^{4}\right)$ it reads

$$
t \simeq t_{2}^{2} /\left(t_{2}-t_{4}\right),
$$

and its fit to "data only" is listed in Table II. The fit is remarkable, given the huge systematic uncertainties, [conservatively, $\pm 5^{0}$ and $5 \%$ error for the $f_{0}(600)$ channel] and we refer to $[8,14]$ for details and a comparison with data. Using Eqs. (1) and (2), the $O\left(p^{6}\right)$ IAM [4,21] reads

$$
t \simeq t_{2}^{2} /\left(t_{2}-t_{4}+t_{4}^{2} / t_{2}-t_{6}\right)
$$

that recovers the $O\left(p^{6}\right) \mathrm{ChPT}$ expansion at low energies and describes well elastic $\pi \pi$ scattering data [21]. In addition, the IAM has a right cut that defines two Riemann sheets. In the second sheet we find poles associated with resonances, in particular, for the $\rho(770)$ in the $(I, J)=(1,1)$ channel and for the $f_{0}(600)$ in the $(0,0)$ one. For narrow resonances, $\Gamma \ll M$, the pole position is related to the mass and width as $\sqrt{s_{\text {pole }}} \simeq M-i \Gamma / 2$, and we keep this as a definition for the wide $f_{0}(600)$, whose $M \sim$ $400-500 \mathrm{MeV}$ and $\Gamma \sim 400-600 \mathrm{MeV}$.

By scaling the previous parameters with their dominant $N_{c}$ behavior, namely, $f_{N_{c}} \rightarrow f \sqrt{N_{c} / 3}, l_{i, N_{c}}^{r} \rightarrow l_{i}^{r} N_{c} / 3$, and $r_{i, N_{c}} \rightarrow r_{i}\left(N_{c} / 3\right)^{2}$, we obtain the large $N_{c}$ dependence of $M_{N_{c}}$ and $\Gamma_{N_{c}}$ for the $\rho$ and $f_{0}(600)$ poles generated by the IAM. If a resonance is predominantly a $\bar{q} q, M_{N} \sim O(1)$, and $\Gamma_{N} \sim O\left(1 / N_{c}\right)$, and so it was shown [7] that the $O\left(p^{4}\right)$ IAM reproduced well that behavior for the $\rho(770)$ and $K^{*}(892)$, two well-established $\bar{q} q$ mesons. This is the expected behavior if in Eq. (3) one neglects the two-meson loop terms, which are subleading in $1 / N_{c}$ with respect to $O\left(p^{4}\right)$ LECS contributions.

In contrast, light scalars follow a qualitatively different behavior. Loop diagrams, instead of the $O\left(p^{4}\right)$ LECS terms, are very relevant in determining their pole position. This is the known fact that light scalars are dynamically generated by the resummation in Eq. (3) of two-meson loop diagrams $[4-6,13,14]$. However, although relevant at $N_{c}=3$, loop diagrams are $1 / N_{c}$ suppressed compared to tree level terms with LECS, and the $O\left(p^{6}\right)$ terms could become bigger at larger $N_{c}$, where the $O\left(p^{4}\right) N_{c}$ results should not be trusted. It is important then to check the $O\left(p^{6}\right)$ IAM: it should give small corrections to the $O\left(p^{4}\right)$ close to $N_{c}=3$, but it may deviate at larger $N_{c}$ and even unveil a subdominant $\bar{q} q$ component.

Before scaling the $O\left(p^{6}\right)$ IAM, let us note that $M_{N_{c}}=$ $O(1)$ and $\Gamma_{N_{c}}=O\left(1 / N_{c}\right)$ is only the leading $\bar{q} q$ scaling. Taking into account subleading orders, to consider a resonance a $\bar{q} q$ state, it is enough that

$$
M_{N_{c}}^{\bar{q} q}=\tilde{M}\left(1+\frac{\epsilon_{M}}{N_{c}}\right), \quad \Gamma_{N_{c}}^{\bar{q} q}=\frac{\tilde{\Gamma}}{N_{c}}\left(1+\frac{\epsilon_{\Gamma}}{N_{c}}\right),
$$

where $\tilde{M}$ and $\tilde{\Gamma}$ are unknown but $N_{c}$ independent and the subleading terms have been gathered in $\epsilon_{M}, \epsilon_{\Gamma}$, which are $O(1)$. Thus, for a $\bar{q} q$ state, the expected $M_{N_{c}}$ and $\Gamma_{N_{c}}$ can be obtained from those at $N_{c}-1$ generated by the IAM,

TABLE I. Sample of LECS central values. The fifth and ninth columns, whose uncertainties roughly represent the previous sample, are used in our fits in the text to calculate $\chi_{\text {LECS }}^{2}$.

\begin{tabular}{cccccccrr}
\hline \hline LECS & $O\left(p^{4}\right)[11]$ & $O\left(p^{4}\right)[17]$ & $O\left(p^{4}\right)[8]$ & $O\left(p^{4}\right)$ we use & $O\left(p^{6}\right)[18]$ & $O\left(p^{6}\right)[16]$ & $O\left(p^{6}\right)[19]$ & $O\left(p^{6}\right)$ we use \\
\hline $10^{3} l_{1}^{r}$ & -6.0 & -5.4 & -3.5 & $-3.5 \pm 2.2$ & -3.3 & -5.2 & -4.6 & $-3.3 \pm 2.2$ \\
$10^{3} l_{2}^{r}$ & 5.5 & 5.7 & 4.7 & $4.7 \pm 1.0$ & 2.9 & 2.3 & 2.0 & $2.9 \pm 1.0$ \\
$10^{3} l_{3}^{r}$ & 0.82 & 0.82 & -2.6 & $0.82 \pm 3.8$ & 1.2 & 0.82 & 0.82 & $0.82 \pm 3.8$ \\
$10^{3} l_{4}^{r}$ & 5.6 & 5.6 & 8.6 & $6.2 \pm 2.0$ & 2.4 & 5.6 & 6.2 & $6.2 \pm 2.0$ \\
\hline \hline
\end{tabular}


TABLE II. Fits to data or constrained to a $\bar{q} q N_{c}$ behavior for the $\rho$ and $f_{0}(600)$. In boldface the $\chi^{2}$ minimized on each fit. For the $O\left(p^{6}\right)$ fits we also provide the $r_{i}$ estimates [16].

\begin{tabular}{lcccccccccccccc}
\hline \hline IAM Fit & $10^{3} l_{1}^{r}$ & $10^{3} l_{2}^{r}$ & $10^{3} l_{3}^{r}$ & $10^{3} l_{4}^{r}$ & $\chi_{\text {data }}^{2}$ & $\chi_{\text {LECS }}^{2}$ & $\chi_{\rho, \bar{q} q}^{2}$ & $\chi_{f_{0}(600), \bar{q} q}^{2}$ & $10^{4} r_{1}$ & $10^{4} r_{2}$ & $10^{4} r_{3}$ & $10^{4} r_{4}$ & $10^{4} r_{5}$ & $10^{4} r_{6}$ \\
\hline$O\left(p^{4}\right)$ Only data & -3.8 & 4.9 & 0.43 & 7.2 & $\mathbf{1 . 1}$ & $\mathbf{0 . 0 8}$ & 0.26 & 140 & \multicolumn{5}{c}{$r_{i}$ estimates from $[16]$ in next row } \\
$O\left(p^{4}\right) \rho$ as $\bar{q} q$ & -3.8 & 5.0 & 0.42 & 6.4 & $\mathbf{1 . 2}$ & $\mathbf{0 . 0 3}$ & $\mathbf{0 . 2 2}$ & 143 & -0.6 & 1.3 & -1.7 & -1.0 & 1.1 & 0.3 \\
$O\left(p^{4}\right) f_{0}(600)$ as $\bar{q} q$ & -3.9 & 4.6 & 2.6 & 15 & $\mathbf{1 . 4}$ & $\mathbf{5 . 6}$ & 0.32 & $\mathbf{1 2 5}$ & & & & & \\
$O\left(p^{6}\right) \rho$ as $\bar{q} q$ & -5.4 & 1.8 & 1.5 & 9.0 & $\mathbf{1 . 1}$ & $\mathbf{1 . 9}$ & $\mathbf{0 . 9 3}$ & 15 & -0.60 & 1.5 & -1.4 & 1.4 & 2.4 & -0.60 \\
$O\left(p^{6}\right) f_{0}(600)$ as $\bar{q} q$ & -5.7 & 2.6 & -1.7 & 1.7 & $\mathbf{1 . 4}$ & $\mathbf{2 . 1}$ & 2.0 & $\mathbf{3 . 5}$ & -0.60 & 1.3 & -4.4 & -0.03 & 2.7 & -0.70 \\
$O\left(p^{6}\right) \rho, f_{0}(600)$ as $\bar{q} q$ & -5.7 & 2.5 & 0.39 & 3.5 & $\mathbf{1 . 5}$ & $\mathbf{1 . 4}$ & $\mathbf{1 . 3}$ & $\mathbf{4 . 0}$ & -0.58 & 1.5 & -3.2 & -0.49 & 2.7 & -0.62 \\
\hline \hline
\end{tabular}

$$
\begin{gathered}
M_{N_{c}}^{\bar{q} q} \simeq M_{N_{c}-1}\left[1+\epsilon_{M}\left(\frac{1}{N_{c}}-\frac{1}{N_{c}-1}\right)\right] \\
\equiv M_{N_{c}-1}+\Delta M_{N_{c}}^{\bar{q} q}, \\
\Gamma_{N_{c}}^{\bar{q} q} \simeq \frac{\Gamma_{N_{c}-1}\left(N_{c}-1\right)}{N_{c}}\left[1+\epsilon_{\Gamma}\left(\frac{1}{N_{c}}-\frac{1}{N_{c}-1}\right)\right] \\
\equiv \frac{\Gamma_{N_{c}-1}\left(N_{c}-1\right)}{N_{c}}+\Delta \Gamma_{N_{c}}^{\bar{q} q} .
\end{gathered}
$$

Note the $\bar{q} q$ index for quantities obtained assuming a $\bar{q} q$ behavior. We refer the values at $N_{c}$ to those at $N_{c}-1$ to be able to calculate from what $N_{c}$ value a resonance starts behaving as a $\bar{q} q$, which is of interest in order to look for subdominant $\bar{q} q$ components. Thus, we can define an averaged $\bar{\chi}_{\bar{q} q}^{2}$ to measure how close a resonance is to a $\bar{q} q$ behavior, using as uncertainty the $\Delta M_{N_{c}}^{\bar{q} q}$ and $\Delta \Gamma_{N_{c}}^{\bar{q} q}$.

$$
\bar{\chi}_{\bar{q} q}^{2}=\frac{1}{2 n} \sum_{N_{c}=4}^{n}\left[\left(\frac{M_{N_{c}}^{\bar{q} q}-M_{N_{c}}}{\Delta M_{N_{c}}^{\bar{q} q}}\right)^{2}+\left(\frac{\Gamma_{N_{c}}^{\bar{q} q}-\Gamma_{N_{c}}}{\Delta \Gamma_{N_{c}}^{\bar{q} q}}\right)^{2}\right] .
$$

Since at $N_{c}=3$ we expect generically $30 \%$ uncertainties, we take $\epsilon_{M}=\epsilon_{\Gamma}=1$. Let us note that $\Delta M$ and $\Delta \Gamma$ tend to zero for large $N_{c}$ and eventually become smaller than our precision determining the pole position, $1 \mathrm{MeV}$, which we add as a systematic error. When a state is predominantly $\bar{q} q$ it should follow Eq. (5) and $\bar{\chi}_{\bar{q} q}^{2} \lesssim 1$. Otherwise, $\bar{\chi}_{\bar{q} q}^{2} \gg 1$. Note that $n$ should not be too far from 3 , since we are looking for the $N_{c}$ behavior of the physical state. If we took $n$ too large, we could be changing radically the original mixture of the observed state and for sufficiently large $N_{c}$ even the tiniest $\bar{q} q$ component could become dominant over the rest [22]. Therefore, our method first determines the behavior of the resonance dominant component, but also, when $\bar{q} q$ is not dominant, the $N_{c}$ at which it becomes so.

Furthermore, by minimizing its $\bar{\chi}_{\bar{q} q}^{2}$ we can constrain a state to follow the $\bar{q} q$ behavior. Thus, we will minimize $\chi_{\text {data }}^{2}+\chi_{\text {LECS }}^{2}$ plus the $\bar{\chi}_{\bar{q} q}^{2}$ of either of the $\rho$, or the $f_{0}(600)$, or both. The averaged $\chi_{\text {LECS }}^{2}$ measures how far the fitted LECS are from their typical values in the tables and stabilizes them. Note that $\pi \pi$ scattering data are poor, with large systematic uncertainties and not very sensitive to some of the individual parameters but to their combinations, thus producing large correlations, driving the LECS away from the typical values for tiny improvements in the $\chi^{2}$, particularly at $O\left(p^{6}\right)$. We will provide the $\chi_{\text {data }}^{2}, \chi_{\text {LECS }}^{2}$, and $\bar{\chi}_{\bar{q} q}^{2}$, divided by the number of data points, the number of LECS and $2 n$, respectively.

Thus, in Table II we show three $O\left(p^{4}\right)$ fits: to data only, constrained to a $\rho \bar{q} q$ hypothesis, or constraining the $f_{0}(600)$ to be a $\bar{q} q$. We list for each fit the different $\chi^{2}$ described above and we see that our approach identifies the $\rho$ as a $\bar{q} q$, since $\bar{\chi}_{\rho, \bar{q} q}^{2} \simeq 0.25$. In contrast, $\bar{\chi}_{\bar{q} q}^{2} \geq 125$ for the $f_{0}(600)$, even if we constrain the fit to minimize also $\bar{\chi}_{\bar{q} q}^{2}$ for the $f_{0}(600)$ (at the price of a higher $\chi_{\text {LECS }}^{2}=5.6$ ). This is the quantitative statement of the $O\left(p^{4}\right)$ results in $[7,8]$ where it was concluded that the main component of the $f_{0}(600)$ was not $\bar{q} q$.

Unfortunately, the $O\left(p^{6}\right)$ analysis has a large freedom and thus $\chi_{\text {LECS }}^{2}$ plays a relevant role to stabilize the fit. In Table II and Fig. 1 we show three $O\left(p^{6}\right)$ fits: constraining the $\rho$ as a $\bar{q} q$ (Fig. 1, top), or the $f_{0}(600)$ (Fig. 1, center) or both (Fig. 1, bottom). As expected, the $O\left(p^{6}\right)$ results are consistent with those at $O\left(p^{4}\right)$ not far from $N_{c}=3[7,8]$ but for the scalar channel they deviate around $N_{c} \sim 8$.

In particular, in the " $\rho$ as $\bar{q} q$ " fit a $\bar{q} q$ dominant nature comes out neatly for the $\rho$, whose $\bar{\chi}_{\bar{q} q}^{2} \sim 0.9$, but is discarded for the $f_{0}(600)$, since its $\bar{\chi}_{\bar{q} q}^{2} \simeq 15$ and Fig. 1 shows that its mass and width both rise when $N_{c}$ increases not too far from real life, $N_{c}=3$. However, for $N_{c}>8$ the mass tends to a constant around $1 \mathrm{GeV}$ and the width decreases, but not with a $1 / N_{c}$ scaling. This suggests a mixing with a $\bar{q} q$ subdominant component, arising as loop diagrams become more suppressed at large $N_{c}$.

One might wonder if the $f_{0}(600)$ could also be forced to behave predominantly as a $\bar{q} q$. Thus we made a " $f_{0}(600)$ as a $\bar{q} q$ " constrained fit (Fig. 1, center) at the price of a worse $\chi_{\text {data }}^{2}$ and an unacceptable $\rho \bar{q} q$ behavior, since its $\bar{\chi}_{\bar{q} q}^{2} \sim 2$. Still, the $f_{0}(600) \bar{\chi}_{\bar{q} q}^{2}$ decreases only to 3.5 (for 34 $N_{c}$ points). This extreme case allows us to conclude that the $O\left(p^{6}\right)$ calculation cannot accommodate a $\bar{q} q$ dominant component for the $f_{0}(600)$.

Finally, we study how much of a subdominant $\bar{q} q$ behavior the $f_{0}(600)$ can accommodate without spoiling that of the $\rho$. Hence, we minimize the $\bar{\chi}_{\bar{q} q}^{2}$ both for the $\rho$ and 

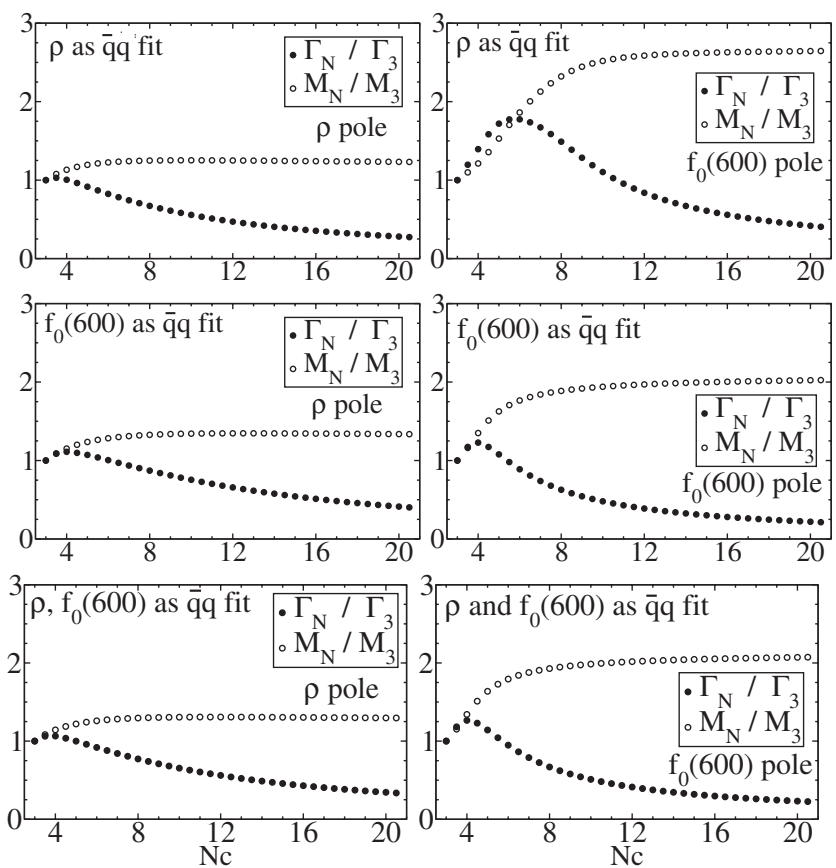

FIG. 1. Mass and width $N_{c}$ behavior of the $\rho$ and $f_{0}(600)$ from an $O\left(p^{6}\right)$ IAM data fit minimizing also the $\bar{\chi}_{\bar{q} q}^{2}$ : of the $\rho$ (top), of the $f_{0}(600)$ (center), of the $f_{0}(600)$ and $\rho$ (bottom).

$f_{0}(600)$ (Fig. 1, bottom). The $f_{0}(600)$ still does not behave predominantly as a $\bar{q} q$, since its $\bar{\chi}_{\bar{q} q}^{2} \simeq 4$. However, it starts behaving as a $\bar{q} q$, i.e., $\bar{\chi}_{\bar{q} q}^{2} \simeq 1$, for $N_{c} \geq 6$. The $\bar{q} q$ behavior of the $\rho$ only deteriorates a little, $\bar{\chi}_{\bar{q} q}^{2} \simeq 1.3$, and should not be pushed much further. This suggests that the subdominant mixing with a $\bar{q} q$ state around $1 \mathrm{GeV}$ seen in the first fit, would become dominant around $N_{c}>6$, at best.

In summary, we have presented a method to determine quantitatively how close the $N_{c}$ dependence of a resonance pole is to a $\bar{q} q$ behavior. We have applied this measure to the poles generated in $\pi \pi$ scattering by unitarized chiral perturbation theory, which is the effective low energy theory of QCD and reproduces systematically its large $N_{c}$ expansion. The method is able to confirm the $O\left(p^{4}\right)$ qualitative results $[7,8]$, identifying the $\rho$ as a $\bar{q} q$ state and showing that the $f_{0}(600)$ is at odds with a dominant $\bar{q} q$ component. We have extended the method to $O\left(p^{6}\right)$ confirming the stability of our $O\left(p^{4}\right)$ conclusions, but also showing that a possible subdominant $\bar{q} q$ may originate around $1 \mathrm{GeV}$. This provides further support, based on the QCD $N_{c}$ dependence, to some models that generate the $f_{0}(600)$ from final state meson interactions, and locate a "preexisting" $\bar{q} q$ scalar nonet $[3,5]$ around $1 \mathrm{GeV}$. The methods presented here should be easily generalized to other dynamically generated mesons [23] and baryons [24].
[1] E. M. Aitala et al. (E791 Collaboration), Phys. Rev. Lett. 86, 770 (2001); Phys. Rev. Lett. 89, 121801 (2002); I. Bediaga and J. M. de Miranda, Phys. Lett. B 633, 167 (2006); M. Ablikim et al. (BES Collaboration), Phys. Lett. B 598, 149 (2004); D. V. Bugg, Phys. Lett. B 572, 1 (2003); 595, 556(E) (2004); Phys. Rep. 397, 257 (2004).

[2] R. L. Jaffe, Phys. Rev. D 15, 267 (1977); Phys. Rev. D 15, 281 (1977); R. Kaminski et al., Phys. Rev. D 50, 3145 (1994); M. Harada et al., Phys. Rev. D 54, 1991 (1996); R. Delbourgo and M. D. Scadron, Mod. Phys. Lett. A 10, 251 (1995); S. Ishida et al., Prog. Theor. Phys. 95, 745 (1996); Prog. Theor. Phys. 98, 621 (1997); N. A. Tornqvist and M. Roos, Phys. Rev. Lett. 76, 1575 (1996); D. Black et al., Phys. Rev. D 58, 054012 (1998); G. Colangelo et al., Nucl. Phys. B603, 125 (2001).

[3] E. Van Beveren et al., Z. Phys. C 30, 615 (1986); E. van Beveren and G. Rupp, Eur. Phys. J. C 22, 493 (2001).

[4] A. Dobado and J. R. Pelaez, Phys. Rev. D 47, 4883 (1993); Phys. Rev. D 56, 3057 (1997).

[5] J. A. Oller and E. Oset, Nucl. Phys. A620, 438 (1997); A652, 407(E) (1999); Phys. Rev. D 60, 074023 (1999).

[6] J. A. Oller et al., Phys. Rev. Lett. 80, 3452 (1998); Phys. Rev. D 59, 074001 (1999); 60, 099906(E) (1999); Phys. Rev. D 62, 114017 (2000); M. Uehara, hep-ph/0204020.

[7] J. R. Pelaez, Phys. Rev. Lett. 92, 102001 (2004).

[8] J. R. Pelaez, Mod. Phys. Lett. A 19, 2879 (2004).

[9] G. 't Hooft, Nucl. Phys. B72, 461 (1974); E. Witten, Ann. Phys. (N.Y.) 128, 363 (1980).

[10] R. L. Jaffe, Proceedings of the International Symposium on Lepton and Photon Interactions at High Energies (University of Bonn, Bonn, Germany, 1981), ISBN .

[11] S. Weinberg, Physica (Amsterdam) 96A, 327 (1979); J. Gasser and H. Leutwyler, Ann. Phys. (N.Y.) 158, 142 (1984); Nucl. Phys. B250, 465 (1985).

[12] T. N. Truong, Phys. Rev. Lett. 61, 2526 (1988); Phys. Rev. Lett. 67, 2260 (1991); A. Dobado et al., Phys. Lett. B 235, 134 (1990).

[13] F. Guerrero and J. A. Oller, Nucl. Phys. B537, 459 (1999); B602, 641(E) (2001).

[14] A. Gómez Nicola and J.R. Peláez, Phys. Rev. D 65, 054009 (2002); AIP Conf. Proc. 660, 102 (2003).

[15] M. Uehara, hep-ph/0308241; hep-ph/0401037; hep-ph/ 0404221.

[16] J. Bijnens et al., Nucl. Phys. B508, 263 (1997).

[17] J. Bijnens et al., Nucl. Phys. B427, 427 (1994).

[18] G. Amoros et al., Phys. Lett. B 480, 71 (2000).

[19] L. Girlanda et al., Phys. Lett. B 409, 461 (1997); J. Nieves and E. Ruiz Arriola, Eur. Phys. J. A 8, 377 (2000).

[20] G. Ecker et al., Nucl. Phys. B321, 311 (1989); J. F. Donoghue et al., Phys. Rev. D 39, 1947 (1989).

[21] J. Nieves et al., Phys. Rev. D 65, 036002 (2002).

[22] Z.X. Sun et al., hep-ph/0503195; J. R. Pelaez, hep-ph/ 0509284.

[23] A. Dobado and J.R. Pelaez, Phys. Rev. D 65, 077502 (2002); L. Roca et al., Phys. Rev. D 72, 014002 (2005).

[24] A. Gomez Nicola et al., Phys. Lett. B 486, 77 (2000). 